科 学 通 报

\title{
嵌段共聚物胶束形成的正电子湮没研究 *
}

\author{
熊兴民 杨巨华 \\ (中国科学院高能物理研究所, 北京 100080)
}

\author{
叶美玲 张小莉 施良和 \\ (中国科学院化学研究所, 北京 100080)
}

\section{关键词正电子晴没、既段共聚物、胶束形成}

嵌段共聚物被誉为高分子合金, 有着广泛的用途. 嵌段共聚物在选择性溶剂中的胶束形成及 其行为多年来引起人们广泛的注意. 尽管至今用了许多实验手段进行了研究, 然而对于这种胶 束生成的微观过程的了解还是很不够的. 正电子湮没技术对溶液中胶束的生成是十分灵敏 的 ${ }^{[1,2]}$. 本文对二嵌段共聚物聚苯乙烯 -二甲基硅氧烷(PSDMS)/正庚烷胶束溶液体系作正电子 湮没寿命参数测量, 研究胶束形成对o- $\mathrm{P}_{\mathrm{s}}$ 生成机率的关系, 为这类嵌段共聚物胶束形成微观机 制提供证据.

\section{1 实 验方 法}

二嵌段共聚物 PSDMS是由美国 Dow Corning 公司提供的, 其重量平均分子量 $\mathbf{M}_{\omega}$ 为 $5.81 \times 10^{4}$, 密度为 $1.4 \mathrm{~g} / \mathrm{cm}^{3}$, 聚苯乙烯嵌段重量百分数为 $23 \%$. 正庚烷溶剂为分析纯. 约 $10 \mu \mathrm{Ci}$ 的 ${ }^{2} \mathrm{NaCl}$ 溶液滴到一片 $3 \mu \mathrm{m}$ 厚的康铜䈃上, 烘干后盖上另一片同样厚度康铜䈃, 然后用粘合剂 将这种夹心源封严. $2 \mathrm{~mL}$ 样品溶液装人特别设计的圆柱样品管内小圆柱套管中. 用一个带 杆的磨口塞将样品管盖严, 并使固定在杆上适当位置的放射源插人样品溶液的正当中. 正电 子寿命谱仪是用 $\mathrm{BaF}_{2}-\mathrm{XP} 2020 \mathrm{Q}$ 闪㷧记数器组装的快慢符合装置 ${ }^{[3]}$, 对 ${ }^{60} \mathrm{Co}$ 源的时间分辨 (FWHM) 为 280 Ps. 对所有不同浓度样品溶液的测量在 室温 $21^{\circ} \mathrm{C}$ 进行. 每个浓度样品测量三 次, 每个谱累积记数为 $1.3 \times 10^{6}$ 以上.

\section{2 结 果 和 讨 论}

用 PC-MEF 计算程序 ${ }^{[4]}$ 作解谱分析. 所有寿命谱能分解成三组分, 最短寿命主要是由于 p-P s 湮没的贡献, 中间寿命归因于正电子与体系中未成束缚态的电子湮没. 上述两个寿命组 分基本上不受溶液浓度变化的影响. 最长寿命组分 $\tau_{3}$ 是由于热化的 o- $\mathrm{P}_{s}$ 的反应和湮没, 其寿 命值在所研究的溶液浓度范围没有观测到有意义的变化 (图 1(a)), 它的相对强度 $I_{3}$ 正比于 o-Ps 生成机率, 该参数对体系的结构和微观环境变化十分灵敏. 正象图 1(b) 结果所显示的那样, 这 些变化能分成 I-III 三个不同的区间. 区间 I 为一个 $I_{3}$ 不随浓度变化的平台 1 , 这时溶液中 $\mathrm{o}-\mathrm{P}_{\mathrm{s}}$ 生成机率保持与溶剂中相同, 表明以自由共聚物形式存在的嵌段共聚物其溶液浓度不影 响体系的 $P_{s}$ 生成. 在区间 I 和 II 之间浓度为 $0.15 \mathrm{mg} / \mathrm{mL}$ 附近 $I_{3}$ 值出现一个陡然下降, 表明

1993-11-29 收稿, 1994-08-01 收修改稿.

*国家自然科学基金和中国科学院核分析开放实验室资助项目. 
自由共聚物分子开始聚集, 它可能是一种具有小聚集数的聚集体, 在较小的浓度范围其数 量随浓度迅速增加. 当浓度增加到一定范围 $(0.56-1.43 \mathrm{mg} / \mathrm{mL})$ 时又出现一个 $I_{3}$ 不随浓度 变化的平台 2 , 这时主要不是聚集体数量的增加而是聚集体中聚集数增加. 在区间 II 和 III 之 间浓度为 $1.43 \mathrm{mg} / \mathrm{mL}$ 附近 $I_{3}$ 进一步出现陡然下降, 表明聚集体结构变化的开始. 可能解释为 当聚集体聚集的分子数增加到某种程度, 聚集体由线状结构转变为更为紧密的球形结构. 这 样可把 $I_{3}$ 的第一和第二个陡然下降对应的浓度分别称为第一和第二临界胶束浓度 $\mathrm{CMC1}$ 和 $\mathrm{CMC}$ (图 1 中插图). 溶液浓度超过 $\mathrm{CMC} 2, I_{3}$ 随浓度增加而逐渐减小主要反映胶束数量随 溶液浓度增加而增加的过程. 在较高的浓度 $(\geqslant 17.7 \mathrm{mg} / \mathrm{mL})$ 呈现出 $I_{3}$ 基本不随浓度变化的 半平台 3. 表明这时胶束的形成和变化对 $P_{s}$ 生成的抑制已达到最大限度.

以往在低分子量的表面活性剂胶束溶液的研究中, o- $\mathrm{P}_{\mathrm{s}}$ 生成机率对溶液浓度的曲线一般 只观测到一个表征临界胶束浓度存在的折断. 认为胶束形成只经历一个由单聚物分子聚集成 具有大聚集数胶束的转变 ${ }^{[1,2]}$. 为什么本工作对嵌段共聚物胶束溶液却观测到两个这样的折

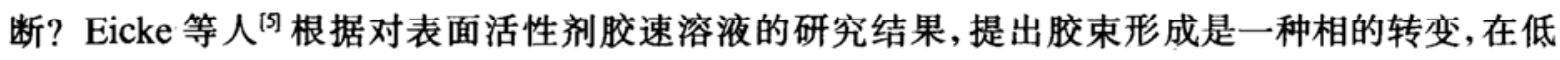

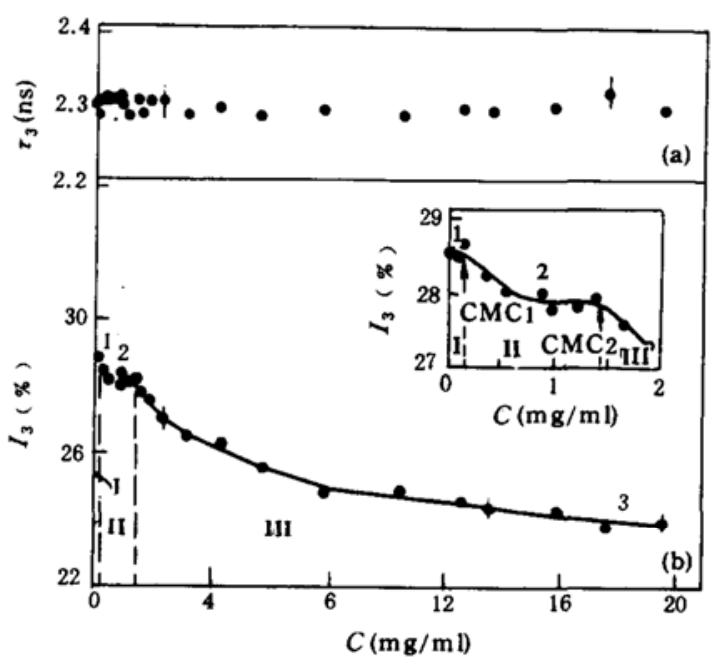

图 1 在 PSDMS/ 正庚烷溶液中 $\tau_{3}$ 和 $I_{3}$ 对浓度的关系

溶液浓度表面活性剂分子以一种初始的线 形排布聚集成预胶束聚集体, 在一定浓度 它能经历一个变成紧密的聚集体的转变. 这个模型被 Fucugauchi 等人 ${ }^{[2]}$ 用来解释 了溶剂对表面活性剂胶束形成的影响. 这样把本工作 $I_{3}-\mathrm{C}$ 曲线的两个折断的 结果分别归因于在嵌段共聚物胶束溶液 中线形聚集体的生成及其向更紧密的球 形聚集体的转变与 Eicke 等人的模型是 一致的. 至今用正电子湮没技术尚未在 表面活性剂胶束溶液中观测到这种线形 预胶束聚集体的生成, 而为什么本工作对 嵌段共聚物 PSDMS 胶束溶液体系却能观 测到? 其原因可能是由于这两种不同类型 的聚合物的分子量和结构的巨大差别. 嵌段共聚物分子量一般为几万到几十万, 比一般只有 几百的表面活性剂分子量大 $2-3$ 个数量级. 这样即使两三个共聚物分子的线形排布的聚 集也有可能形成较紧密的聚集体, 产生有别于在表面活性剂溶液中形成这样的线形预胶束聚 集体的变化, 从而对 $\mathrm{P}_{\mathrm{s}}$ 生成产生显著的影响. 此外, 由于所研究的嵌段共聚物的大分子链和 该共聚物链中苯环的空间效应, 可能使得有较小聚集数的线形聚集体产生进一步聚集态结构 变化不太容易, 需要更多的共聚物分子的聚集来产生更大的聚集力克服这些空间效应, 使聚 集体结构发生变化, 因而这种线形聚集体有一个相当明显的形成阶段. 如上述 o-P $\mathrm{P}_{\mathrm{s}}$ 生成机率不 随浓度变化的平台 2 似乎就是反映这种线形聚集体聚集数增加的过程.

根据对表面活性剂胶束溶液的研究提出的达到热化前高能的或 “热” 的正电子或正 电子素原子 (以下称为热化 $o-P_{s}$ 前身) 能与溶质 (胶束) 反应的热化 $o-P_{s}$ 前身反应模型 ${ }^{[1,6}$, 认为 在嵌段共聚物胶束溶液中, 热化的 o-P $\mathrm{P}_{s}$ 前身的总产额等于在纯溶剂寿命谱中长寿命组分的强 
度 $I_{3}^{0}$, 其中一部分不与溶质反应, 其产额为 $I_{3}^{m}$, 另一部分产额为 $I_{3}{ }^{\prime}$ 的 o-P $\mathrm{P}_{s}$ 前身 与溶质反应, 因 此有

$$
I_{3}^{0}=I_{3}^{n}+I_{3}^{r},
$$

由实验结果 $\mathrm{o}-\mathrm{P}_{\mathrm{s}}$ 寿命 $\tau_{3}$ 在研究的溶液浓度范围为常数值 (图 1(a)), 在浓度超过临介胶束浓度 $(\mathrm{CMC} 2)$ 时 o- $\mathrm{P}_{\mathrm{s}}$ 强度 $I_{3}$ 随浓度增加而减小, 表明胶束是 o- $\mathrm{P}_{\mathrm{s}}$ 的抑制剂, 则抑制对浓度的关系能以下式来描述 ${ }^{[7}$ :

$$
I_{3}=I_{3}^{n}+I_{3}^{r} /(1+K C) \text {, }
$$

$K$ 为抑制常数, $C$ 为溶液浓度. 由 (1) 和 (2) 式得

$$
\left(I_{3}^{0}-I_{3}\right) / C=K_{3}-K_{3}^{n} \text {. }
$$

表 1 PSDMS/正庚烷溶液和表面活性剂溶液的抑制常数 和不被清扫分数

\begin{tabular}{c|c|c|c|c|c}
\hline 样品体系 & 溶 质 & 溶剂 & $I_{3}^{n} / I_{3}^{0}$ & $K(\mathrm{~mol} / \mathrm{L})$ & 参考文献 \\
\hline 嵌段共聚物溶液 & PSDMS & 正庚熀 & 0.78 & 9446 & 本工作 \\
\hline \multirow{3}{*}{ 表面活性剂溶液 } & $\mathrm{DAB}$ & 环乙烧 & 0.71 & 518 & \\
& $\mathrm{DAB}_{Z}$ & 环乙烷 & 0.64 & 852 & {$[7]$} \\
& $\mathrm{AOT}$ & 并辛烷 & 0.80 & 5662 & \\
\hline
\end{tabular}

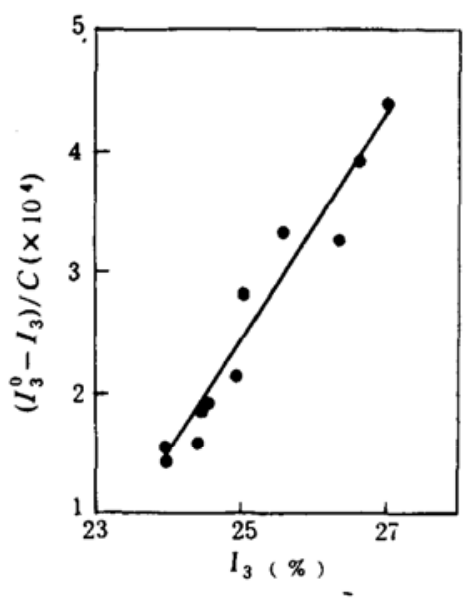

图 2 在 PSDMS/ 正庚烷溶液中热 化 o-Ps 前身反应模型

用图 1 中浓度大于 $\mathrm{CMC} 2$ 的数据以 $\left(I_{3}^{0}-I_{3}\right) / C$ 对 $I_{3}$ 作图, 从图 2 看到 $\left(I_{3}^{0}-I_{3}\right) / C$ 对 $I_{3}$ 是很好 的直线关系. 通过最小二乘拟合, 由直线的斜率 $K$ 和截距 $-K I_{3}^{n}$ 得到抑制常数 $K=9446 \mathrm{~mol} / \mathrm{L}^{-1}$, 不被清扫的 o- $P_{s}$ 前身的分数 $I_{3}^{n} / I_{3}^{0}=0.78$. 从表 1 可见, 本工作嵌段共聚物 PSDMS-33/ 正庚烷溶 液的 $I_{3}^{n} / I_{3}^{0}$ 值与几种表面活性剂胶束溶液的值基本相同, 表明嵌段共聚物溶液中的胶束与表 面活性剂溶液中的胶束一样能与 “热”的正电子或正电子素原子反应, 对 o-Ps 有基本相同的抑 制作用. 根据 $P_{s}$ 优先与分子中的活性中心反应而不是与整个分子 ${ }^{[7]}$, 由于在大的嵌段共聚物分 子链中比在低分子量的表面活性剂分子链中可能存在着更多的活性中心, 因此以克分子浓度 的倒数表示的 $K$ 值与表面活性剂比较, 所得的嵌段共聚物胶束溶液的 $K$ 值大得多是热化o- $\mathrm{P}_{\mathrm{s}}$ 前身反应模型应用的合理结果. 上述结果表明热化 $0-\mathrm{P}_{\mathrm{s}}$ 前身的反应模型能较好地描述嵌段共 聚物溶液体系胶束形成对 o- $\mathrm{P}_{\mathrm{s}}$ 的抑制. 本工作对嵌段共聚物胶束溶液的研究给出了胶束形成 的微观过程的信息, 观测到以往在低分子量的表面活性剂胶束溶液中未能观测到的胶束形成 的两阶段过程.

\section{参考文献}

[1] Jean, Y. C., Ache, H. J., J. Am. Chem. Soc., 1978, 100:6320.

[2] Fucugauchi, L. A. et al., J. Am. Chem. Soc., 1978, 100:2851.

[3] Chang Tianbao et al., Nucl. Inst. and Meth., 1978, A256:398.

[4] 熊兴民, 核技术, 1983, (5): 11 .

[5] Eicke, H. F. et al., in Micellization, Solubilization and Microemulsions (ed. Mittal, K. L.), Plenum Press, New York, 1977, 11:429.

[6] Alfassi, Z. B., Ache, H. J., J. Phys. Chem., 1984, 88: 4347.

[7] Levay, B., Atomic Energy Review, 1979, 17:2. 